OPEN ACCESS

Edited by:

Feng Liu,

Tianjin Medical University General Hospital, China

Reviewed by: Meiling Li,

Harvard Medical School,

United States

Xin Di,

New Jersey Institute of Technology,

United States

*Correspondence:

Guihua Jiang

jianggh@gd2h.org.cn

Specialty section:

This article was submitted to Neuroimaging and Stimulation, a section of the journal

Frontiers in Psychiatry

Received: 23 October 2018

Accepted: 28 March 2019

Published: 12 April 2019

Citation:

Fu S, MaX, Wu Y, Bai Z, Yi Y,

Liu M, Lan Z, Hua K, Huang S, Li M and Jiang G (2019) Altered Local and Large-Scale Dynamic Functional Connectivity Variability in Posttraumatic Stress Disorder:

A Resting-State fMRI Study.

Front. Psychiatry 10:234.

doi: 10.3389/fpsyt.2019.00234

\section{Altered Local and Large-Scale Dynamic Functional Connectivity Variability in Posttraumatic Stress Disorder: A Resting-State fMRI Study}

\author{
Shishun $\mathrm{Fu}^{1,2}$, Xiaofen $\mathrm{Ma}^{2}$, Yunfan $\mathrm{Wu}^{2}$, Zhigang $\mathrm{Bai}^{3}$, Yin $\mathrm{Yi}^{1,2}$, Mengchen Liu ${ }^{1,2}$, \\ Zhihong Lan ${ }^{1,2}$, Kelei Hua ${ }^{1,2}$, Shumei Huang ${ }^{2,4}$, Meng Li $^{2}$ and Guihua Jiang ${ }^{2,1 *}$
}

${ }^{1}$ The Second School of Clinical Medicine, Southern Medical University, Guangzhou, China, ${ }^{2}$ The Department of Medical Imaging of Guangdong Second Provincial General Hospital, Guangzhou, China, ${ }^{3}$ The Department of Medical Imaging of Affiliated Hospital, Inner Mongolia University for Nationalities, Hohhot, China, ${ }^{4}$ Guangdong Medical University, Dongguan, China

Posttraumatic stress disorder (PTSD) is a psychiatric condition that can emerge after exposure to an exceedingly traumatic event. Previous neuroimaging studies have indicated that PTSD is characterized by aberrant resting-state functional connectivity (FC). However, few existing studies on PTSD have examined dynamic changes in resting-state FC related to network formation, interaction, and dissolution over time. In this study, we compared the dynamic resting-state local and large-scale FC between PTSD patients $(n=22)$ and healthy controls ( $\mathrm{HC} ; n=22$; conducted as standard deviation in resting-state local and large-scale FC over a series of sliding windows). Local dynamic FC was examined by calculating the dynamic regional homogeneity (dReHo), and large-scale dynamic FC (dFC) was investigated between regions with significant dReHo group differences. For the PTSD patients, we also investigated the relationship between symptom severity and dFC/ dReHo. Our results showed that PTSD patients were characterized by I) increased dynamic (more variable) dReHo in left precuneus (PCu); II) increased dynamic (more variable) dFC between the left PCu and left insula; and III) decreased dFC between left PCu and left inferior parietal lobe (IPL), and decreased dFC between left PCu and right PCu. However, there is no significant correlation between the clinical indicators and dReHo/dFC after the family-wise-error (FWE) correction. These findings provided the initial evidence that PTSD is characterized by aberrant patterns of fluctuating communication within brain system such as the default mode network (DMN) and among different brain systems such as the salience network and the DMN.

Keywords: posttraumatic stress disorder, resting-state functional magnetic resonance imaging, dynamic functional connectivity, regional homogeneity, default mode network

\section{INTRODUCTION}

Posttraumatic stress disorder (PTSD) is a psychiatric condition that can emerge after exposure to an exceedingly traumatic event (1). In the general population, PTSD occurs most commonly after traffic accidents and affects $10 \%-32 \%$ of those involved within 12 months after the event (2). Symptoms of PTSD include intrusive memories, hypervigilance, insomnia, and emotional 
numbing (3). Previous studies have indicated that PTSD patients exhibited abnormal interactions among the brain systems $(4,5)$. For example, Zhang et al. found that the dorsolateral prefrontal cortex showed increased resting-state functional connectivity (FC) with the visual cortex, suggesting that the disrupted frontaloccipital system may be associated with the dysfunction of visual information processing (5). One of robustly identifiable networks is the default mode network (DMN) (6), which is involved in processing self-relevant stimuli $(7,8)$. The dysfunction of the DMN in PTSD patients may indicate impaired self-generated thoughts and autobiographical memory during rest (9).

One effective approach for exploring brain communication is through the analysis of resting-state fMRI studies (10). Recent resting-state studies in both animals and humans have revealed the dynamic nature of the spatiotemporal organization of blood oxygen level-dependent (BOLD) signals (11-13). Due to unconstrained mental activity, the resting state even shows more dynamic features than in task-stimuli studies (14). A recent study of dynamic FC network indicated that the static FC represented average connectivity across different dynamic states during the whole scanning period; it may not be sensitive enough to detect the alteration of neurofluctuations (15). In order to investigate the dynamic features of inter-regional BOLD signal fluctuations over temporal scales, the sliding window analysis of dynamic FC (dFC) was developed. This approach measured the variety correlations among discrete (large-scale) brain regions (16) using a short, sliding temporal window. Kaiser et al. found that the resting-state $\mathrm{dFC}$ revealed the interactions among networks or subnetworks over time (17). Early studies suggested that the $\mathrm{dFC}$ can be associated with the changes in arousal (18) and vigilance (19) since hypervigilance and hyperarousal are two typical symptoms of PTSD. We proposed to use $\mathrm{dFC}$ to investigate the characteristic features of PTSD in the resting state. Moreover, since changes in brain network topology are associated with those in local brain activity (20), it was reasonable for us to measure both the large-scale and local dynamic FC in our study.

Regional homogeneity ( $\mathrm{ReHo}$ ) is one of the commonly used algorithms in measuring local FC (21-23). ReHo is a reliable measurement technique and robust against noise in the fast imaging sequence data (24). A prior animal study has suggested an association between ReHo variability and different states of neural activity (25). A recent study of dReHo using the sliding-window approach also indicated that brain regions with high dReHo fluctuation tended to be functional hubs in brain systems (26). A resting-state study has shown that the gene variants affected dReHo in attention-deficit/hyperactivity disorder (27). These findings introduced the clinical potential of dReHo analysis.

The investigation of $\mathrm{dFC}$ and $\mathrm{dReHo}$ in the resting state may provide new insight into the aberrant brain connectivity in PTSD. Previous studies investigating major depression (28), schizophrenia $(29,30)$, and bipolar disorder (31) showed abnormal $\mathrm{dFC}$ and $\mathrm{dReHo}$ under the resting state of these patients, and all of these psychiatry researches found aberrant $\mathrm{dFC}$ or aberrant dynamic local activities in the DMN. A possible explanation for these abnormalities is the dynamic nature of the DMN, which exhibits dynamic interactions with a number of other brain systems in the resting state (32). Kaiser et al. (17) indicated that the investigation of altered dynamic activity in areas of the DMN may be important in understanding the pathophysiology of psychiatric disorders.

Although there are few studies available that have focused on the dynamic brain activity in PTSD, prior static studies have suggested that the symptoms of PTSD are associated with the DMN. Mounting evidence has indicated that PTSD is associated with aberrant DMN connectivity (33-35). A static FC ( $\mathrm{sFC}$ ) study suggested that the aberrant activities in the DMN can be a predictor of the symptom severity of PTSD (8). A previous study also approved that the static fMRI data can be used to discriminate the PTSD from HC by using the multilevel parametric classification approach (36). A recent study compared the accuracy of sFC to the accuracy of dFC in classifying PTSD patients and HC (37). The results showed that the peak classification accuracy of dFC reached $94.2 \%$, while the peak classification accuracy of sFC was $86.7 \%$; this research indicated that the temporal $\mathrm{dFC}$ is a better predictor than sFC of the diagnostic features of PTSD. Additionally, this study indicated that, in comparison with the HC, the PTSD patients were characterized by decreased temporal variability of brain connectivity. Preti et al. also indicated that PTSD patients often stay trapped in one state and exhibited a decreased dFC in comparison with $\mathrm{HC}$ subjects (38). All of these studies indicated that the aberrant connectivity variability of brain networks is vital in the investigation of the neurophysiological mechanism of PTSD.

In order to explore the characteristic resting-state temporal variability of PTSD, we decided to measure both large-scale and local dynamic FC. Based on previous findings, we hypothesized that PTSD patients would exhibit altered dReHo in regions within the DMN. We also expected regions with dReHo alterations to show aberrant $\mathrm{dFC}$ and the connectivity measures to be associated with subjects' symptomatology.

\section{METHOD}

\section{Subjects}

Permission to undertake this study was granted by the ethics committee of Guangdong Second Provincial General Hospital. In January and February 2017, we recruited 30 trauma-exposed subjects from a serious highway traffic accident in Guangdong province. Prior to the examination, none of the patients had undergone any psychotherapy. The inclusion criteria for the PTSD patients were as follows: I) age $>18$ years; II) right-hand dominance; III) no preexisting psychiatric disorders or physical conditions as determined by a structural clinical interview using the Diagnostic and Statistical Manual of Mental Disorders, 4th edition (DSM-IV); IV) no psychiatric medications or substance abuse; V) no MR imaging contraindications; VI) no head trauma or neurologic disorders; VII) fulfills the criteria of DSM-IV and has a ClinicalAdministered PTSD Scale (CAPS) score >40; and VIII) not pregnant or nursing. After considering the strict requirements, eight subjects were excluded, five of them for failing to obtain the 
CAPS score $>40$. Twenty-two demographically matched healthy controls (HCs) were recruited for this study. The inclusion criteria for HCs were as follows: I) age $>18$ years; II) right-hand dominance; III) no preexisting psychiatric disorders or physical conditions as determined by a structural clinical interview using the DSM-IV; IV) no psychiatric medications or substance abuse; V) no MR imaging contraindications; and VI) not pregnant or nursing. Each participant provided written informed consent, which was obtained prior to the MRI scanning.

\section{Assessment of Mental Status}

PTSD diagnosis was determined following the DSM-IV diagnostic criteria. Before undergoing resting-state MRI, all PTSD patients were screened with CAPS (39) in order to estimate the intensity and frequency of the symptoms. In addition, emotion assessments were conducted of all participants, including the Self-rating Anxiety Scale (SAS) (40) and the Selfrating Depression (SDS) (41), in order to evaluate the emotional status. A further Structured Clinical Interview for DSM-IV was also performed to evaluate psychiatric disorder comorbidities.

\section{Magnetic Resonance Imaging Data Acquisition}

Each of the participants underwent a resting-state MRI in a 3.0-T MR imager (Ingenia; Philips, Best, The Netherlands) equipped with a 32-channel head coil at the Department of Medical Imaging in Guangdong Second Provincial General Hospital. A diagnostic T1-weighted image and a T2 fluid attenuated inversion recovery (T2-FLAIR) image were taken to exclude participants with brain lesions. The resting-state fMRI data were acquired using gradient echo-planar imaging (EPI) with the following parameters: repetition time (TR)/echo time $(\mathrm{TE})=2,000 \mathrm{~ms} / 30 \mathrm{~ms}$; matrix $=64 \times 64$; field-of-view $=230 \mathrm{~mm} \times 230 \mathrm{~mm}$; flip angle $=90$; slice thickness $=3.6 \mathrm{~mm}, 0.6-\mathrm{mm}$ gap; interleaved scanning; 38 transverse slices covering the whole brain at all 240 volumes were acquired for each participant within $480 \mathrm{~s}$; each volume was aligned along the anterior-posterior commissure. Each participant was instructed to lie still and to avoid falling asleep or thinking of anything in particular during MR scanning.

\section{Resting-State Functional Magnetic Resonance Imaging Data Preprocess}

Standard preprocessing of the functional images was performed with the DPARSF 4.3 Advanced Edition (http://rfmri.org/ DPARSF) and the SPM12 package (www.fil.ion.ucl.ac.uk/spm) based on MATLAB (Mathworks, Inc., Natick, MA, USA). The first 10 volumes of each dataset were discarded for signal equilibration. The remaining data were performed using slice timing correction and realignment and co-registered with the anatomical scan. The co-registered T1-weighted images were segmented into gray matter, white matter, and cerebrospinal fluid. And then the functional images were normalized into the Montreal Neurological Institute $(\mathrm{MNI})$ space with a voxel size of $3 \times 3 \times 3 \mathrm{~mm}^{3}$. The head movement parameters were obtained from the realignment steps in the DPARSF. We took the mean FD Jenkinson (42) as the head motion
TABLE 1 | Demographic and clinical data.

\begin{tabular}{lcccc}
\hline Characteristic & PTSD $(\boldsymbol{n}=\mathbf{2 2})$ & HC $(\boldsymbol{n}=\mathbf{2 2})$ & $\boldsymbol{t}$ value & $\boldsymbol{P}$ value \\
\hline Age (years) & $37.36 \pm 8.95$ & $40.32 \pm 10.34$ & -1.014 & 0.317 \\
Gender (M/F) & $8 / 14$ & $8 / 14$ & & \\
Head motion & $0.169 \pm 0.443$ & $0.159 \pm 0.441$ & 0.073 & 0.942 \\
Education (years) & $11.82 \pm 3.22$ & $10.45 \pm 4.25$ & 1.200 & 0.237 \\
CAPS & $51.45 \pm 6.93$ & & & \\
SAS & $36.09 \pm 8.11$ & $38.18 \pm 6.02$ & -0.971 & 0.337 \\
SDS & $38.05 \pm 9.49$ & $39.09 \pm 8.08$ & -0.393 & 0.696
\end{tabular}

Demographic data are presented as mean $\pm S D$. PTSD, posttraumatic stress disorder; HC, healthy control; CAPS, Clinical-Administrated PTSD Scale; SAS, Selfrating Anxiety Scale; SDS, Self-rating Depression Scale. The P-value was obtained by the two-sample t test.

reference standard. We eliminated the subjects with motion (mean FD Jenkinson) greater than $2 \times$ standard deviation (SD) above the group mean motion as recommended in a previous study (43). No subject was eliminated in this step. There was no significant difference in head motion between the PTSD patients and the HC (see Table 1). Linear detrending processing was conducted to remove the linear signal drift. Individual-level regression analysis was conducted to minimize the influence of head motion (Friston 24 model), white matter signal noise, and cerebrospinal fluid signal noise. A temporal band-pass filter $(0.0167-0.10 \mathrm{~Hz})$ was applied to the data to remove the physical noise and any frequencies for which the period was shorter than that of a single sliding window (44). We performed spatial smoothing with a 6-mm full-width at halfmaximum (FWHM) kernel before performing the dReHo group analysis. As for the dFC, we performed the spatial smoothing with a 6-mm FWHM kernel before the linear detrending and nuisance signals regression, and band-pass filtering. Considering the size of FWHM Gaussian kernel might affect the results of $\mathrm{dReHo} / \mathrm{dFC}$ analysis $(45,46)$, we used 4- and 8-mm FWHM Gaussian kernel to test the consistency of our results $(45,46)$ (see Supplementary Figures 1 and 2).

\section{Computation of dReHo and dFC}

ReHo calculation: The ReHo algorithm measures voxel-wise short-distance FC with Kendall's coefficient of concordance (23) using the following formula:

$$
W=\frac{\sum_{i=1}^{N} R_{i}^{2}-N \bar{R}^{2}}{\frac{1}{12} K^{2}\left(N^{3}-N\right)}
$$

where $W$ is the Kendall's coefficient of concordance among the given voxels, $N$ denotes the length of the time series, $K=27$ is the size of the voxel cluster containing $3 \times 3 \times 3$ adjacent voxels, $R_{i}$ denotes the summation of the rankings of the BOLD signal amplitude of all $K$ voxels at the $i$ th time point, and $R$ is the mean of $R_{i}$.

To compute the dReHo for these data, the time course was segmented into 60-s Hamming windows (30 dynamics). By sliding the onset of each window by 2 dynamics (4s), for a total of 101 overlapping windows in the first level analysis, the dReHo was estimated by using the calculated SD of the ReHo 
through the windows at each voxel, yielding a set of ReHo maps for each participant.

Two-sample $t$-test with head motion parameters (mean FD Jenkinson values), age, and sex as covariates was performed to test the difference in dReHo maps between the PTSD patients and HC at each voxel. Multiple comparisons correction was performed with Gaussian random field (GRF) theory at the cluster level (minimum $z>3.54$; cluster significance: $p<0.05$, two-tailed GRF corrected).

To determine whether the dReHo metrics were associated with clinical indicators, we performed general linear models with the clinical indicators (CAPS, SAS, SDS) and mean dReHo values from clusters with significant group differences as independent variables, and head motion parameters, age, and sex as covariates. The correlation analysis was accomplished with the SPSS software with a significance threshold of $p<0.05$ (uncorrected).

Voxel-wise seed-based FC analyses were performed using the DPARSF 4.3. We employed the aberrant dReHo region, which we calculated above, as a seed region. Then we used the slidingwindow approach as we have used in the dReHo calculation; the time course was segmented into 60 -s Hamming windows by sliding the onset of each window by 2 dynamics, for a total of 101 overlapping windows in the first level analysis. Within each sliding window, the whole brain FC maps for the seed region were computed as the Fisher $z$ transformed Pearson correlation coefficient between the averaged time course of all voxels in the seed and the time course of all other voxels in the whole brain, yielding a set of sliding window $z \mathrm{FC}$ maps for each participant. The $\mathrm{dFC}$ was estimated by calculating the standard deviation in $z \mathrm{FC}$ values through windows at each voxel.

Two-sample $t$-test with head motion parameters (mean FD Jenkinson values), age, and sex as covariates was performed to investigate the difference of $\mathrm{dFC}$ values between the PTSD group and the HC group at each voxel. Multiple comparisons were performed with GRF correction at the cluster level (minimum $z>3.29$; cluster significance: $p<0.05$, two-tailed GRF corrected).

To explore the relationship between $\mathrm{dFC}$ metrics and clinical indicators, we performed general linear models with the clinical indicators and mean $\mathrm{dFC}$ values from clusters with significant group differences as independent variables, and head motion parameters, age, and sex as covariates. The correlation analysis was accomplished with SPSS software with significance threshold of $p<0.05$ (uncorrected).

\section{RESULTS}

The demographic and clinical data are summarized in Table $\mathbf{1 .}$ Inconsistent with our prediction, compared with the $\mathrm{HC}$, the PTSD patients exhibited an increased dReHo (more variability) in the left posterior cingulate cortex $(\mathrm{PCC}) /$ precuneus $(\mathrm{PCu})$ (Figure 1). We also found a decreased dFC (less variability) between the left and right $\mathrm{PCu}$, and the left inferior parietal lobe (IPL)/angular gyrus (AG), but increased dFC between the PCC and the left insula (Table 2 and Figure 2).

Next, we conducted analyses to test the association between the clinical indicators (CAPS, SAS, SDS) of PTSD and dReHo within the PTSD group. There is no significant correlation between the clinical indicators and $\mathrm{dReHo} / \mathrm{dFC}$ after $\mathrm{FWE}$ correction (see Supplementary Figure 4).

\section{DISCUSSION}

In this study, by using resting-state dReHo analysis, we determined the local aberrant variability in the left $\mathrm{PCu}$. The $\mathrm{PCu}$ is a key hub in the DMN of human brain (47). Aberrant restingstate temporal dynamic brain activities were found in the $\mathrm{dReHo}$ and large-scale $\mathrm{dFC}$ of specific brain regions, which were mainly located in the posterior $\mathrm{DMN}(\mathrm{pDMN})$ and the primary region of the salience network (SN). These observations provide new insights into the aberrant brain activities in PTSD.

This study revealed a significantly increased dReHo (more variability) in left $\mathrm{PCu}$, suggesting that the neurofluctuation of the left PCu is unstable in the PTSD patients in the resting state. The PCu is a key hub of the pDMN (47-49) and is considered to

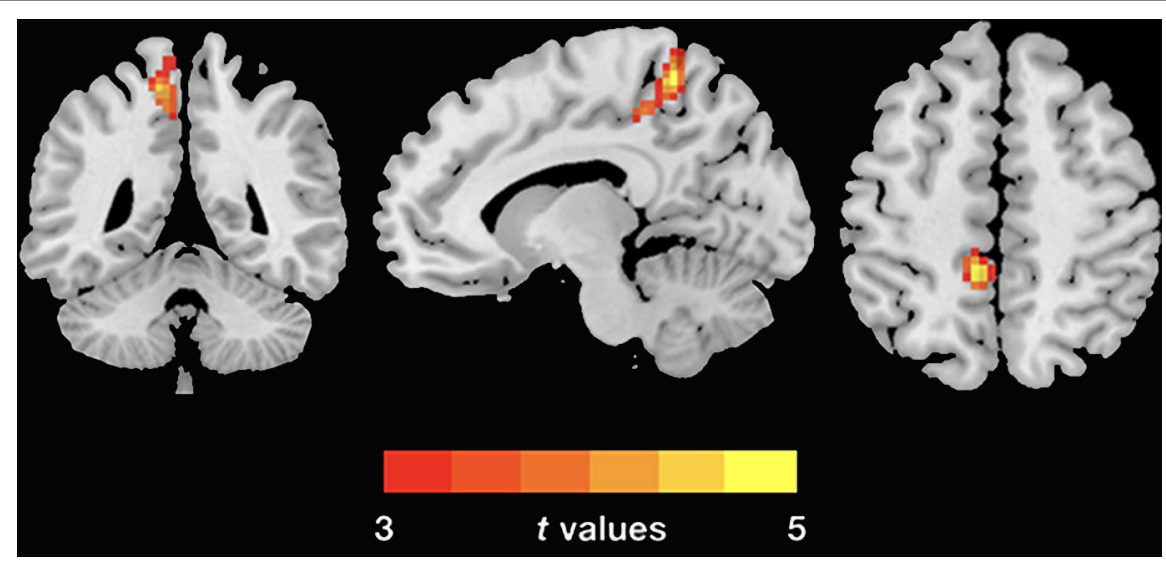

FIGURE 1 | Group differences of dReHo variability were revealed by two-sample $t$-test. The PTSD group shows increased (warm color) dReHo variability in the left PCu (cluster size: 104; AAL: Precuneus_L; Brodmann area 7; MNI coordinates: X: -12 Y: -48 Z: 60 ; peak $t$-value = 5.1868) relative to the HC. The Gaussian random theory was used for cluster-level multiple comparison correction (minimum $z>3.54$; cluster significance $p<0.05$, GRF corrected). dReHo, dynamic regional homogeneity; PTSD, posttraumatic stress disorder; AAL, anatomical automatic labeling; PCu, precuneus; MNI, Montreal Neurological Institute. 
TABLE 2 | Comparison of dFC between PTSD and HC.

\begin{tabular}{|c|c|c|c|c|c|c|c|}
\hline \multirow[t]{2}{*}{ Brain region } & \multirow[t]{2}{*}{ Cluster size } & \multicolumn{3}{|c|}{ MNI coordinates } & \multirow[t]{2}{*}{ AAL } & \multirow[t]{2}{*}{ Brodmann's area } & \multirow{2}{*}{$\begin{array}{c}\text { Peak } \\
t \text { value }\end{array}$} \\
\hline & & $\mathbf{x}$ & $\mathbf{Y}$ & $\mathbf{Z}$ & & & \\
\hline $\mathrm{RPCu}$ & 44 & 3 & -48 & 45 & Precuneus_R & 7 & -4.0992 \\
\hline L IPL & 62 & -38 & -78 & 42 & Parietal_Inf_L & 19 & -4.1411 \\
\hline L Insula & 33 & -36 & -12 & 21 & Insula_L & 13 & 4.6200 \\
\hline
\end{tabular}

L, left; R, right; MNI, Montreal Neurological Institute; dFC, dynamic functional connectivity; AAL, anatomical automatic labeling; PCu, precuneus; IPL, inferior parietal lobe.

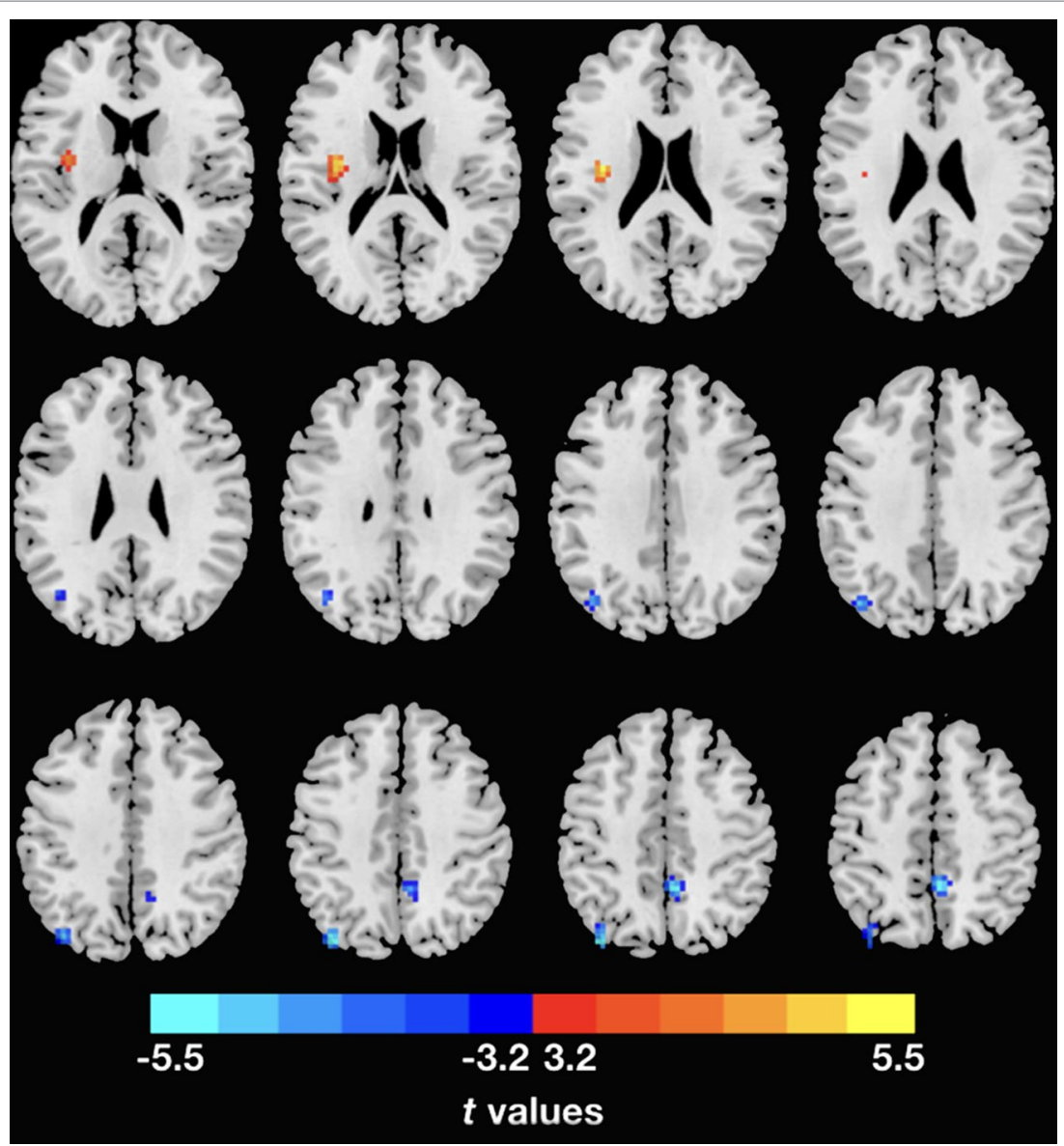

FIGURE 2 | Inter-regional dFC differences with regions showing significant dReHo differences between groups. The PTSD group shows increased dFC variability in left insula, but decreased dFC variability in right PCu and left IPL relative to the HC. Gaussian random theory was used for cluster-level multiple comparison correction (minimum z > 3.29; cluster significance $p<0.05$, GRF corrected). dFC, dynamic functional connectivity; dReHo, dynamic regional homogeneity; PTSD, posttraumatic stress disorder; PCu, precuneus; IPL, inferior parietal lobe.

be involved in self-referential processing (7) and autobiographic memory (50). In the resting state, the DMN exhibits dynamic interaction with a number of brain systems, such as the frontalparietal control network and the dorsal attention network (32). Although we found an aberrant local connectivity variety in the PCu, the evidence of aberrant DMN connectivity in PTSD patients is not entirely persuasive. In order to explore the dynamic interactions among the DMN and other brain networks, we employed the left $\mathrm{PCu}$ as the seed region and carried out the $\mathrm{dFC}$ of the whole brain.
Several studies have identified aberrant activities in the DMN of PTSD patients $(6,34,35)$. In the present study, using seedbased $\mathrm{dFC}$, we identified lower-variability regions located in the right $\mathrm{PCu}$ and the left IPL. These results suggest that compared with the normal controls, the PTSD group exhibits decreased dFC (less variability) within the pDMN. We suspected that this restrained neurofluctuation within the pDMN represents decreased regulation of the self-referential processing. Previous studies using the independent component analysis identified the aberrant $\mathrm{pDMN}$ in PTSD patients $(4,51)$. Furthermore, 
Zhang et al. found a decreased intranetwork connectivity within the $\mathrm{pDMN}$ by measuring resting-state sFC. These researches suggested that the decreased $\mathrm{FC}$ in $\mathrm{pDMN}$ was associated with the dysfunction of evaluation of the self-related events in PTSD. The interaction between DMN and the executive control systems, which includes dorsal lateral middle frontal cortex and the IPL (52), is essential in regulating the self-generated thought (32). In the present study, a deceased dFC was found between the left PCu and the left IPL. Therefore, we inferred that the deceased dFC suggested a discrete FC state between the DMN and the executive control system, which may induce the dysregulation of self-referential processing. Previous studies indicated that the IPL is engaged in mediating visuospatial processing (53), which is critical when dealing with life-threatening events (54). Additionally, previous study indicated that the IPL is a vulnerable brain region to the neurotoxic effects of stress (55). Therefore, we suspected that the aberrant $\mathrm{dFC}$ between the PCu and the IPL might be a potential biomarker of PTSD.

In the present study, the left insula was the only region in the brain that exhibited significant positive dynamic correlation with the left PCu in the PTSD group. The insula is a key hub in the $\mathrm{SN}$ and is thought to be involved in the detection of personally salient internal and external stimuli that guide behaviors in order to maintain equilibrium (56). In addition, the insula is thought to be involved in mediation of the "switching" between activation of the DMN and the central executive network (CEN) to direct appropriate behavioral responses to the salience stimuli (57). Therefore, we suspected that the positive dynamic correlation between the left PCu and left insula might suggest an excessive interaction between the SN and the DMN. Previous studies using the graph theory approach identified dysregulation in three intrinsic brain networks $(1,58)$. Lei et al. found a disequilibrium among the CEN, DMN, and SN and suggested that the SN was crucial to the PTSD symptoms (58). Previous resting-state sFC study also revealed an increased correlation between the DMN seed region [PCC and ventromedial prefrontal cortex (vmPFC)] and SN (insula and precentral sulcus) (59). They found a positive correlation between the PTSD symptom severity and the vmPFC-precentral sulcus FC values. Our results provided an additional piece of evidence that, compared with the HC, the PTSD patients exhibited more variable connectivity between the DMN and the SN.

There are some limitations in the present study that should be highlighted. Firstly, little information is available on the meaning of the resting-state $\mathrm{dFC}$ in neurocognitive functioning. For example, it remains unknown whether the abnormal dynamic activities in the resting state are intrinsic properties or

\section{REFERENCES}

1. Yehuda R, Hoge CW, McFarlane AC, Vermetten E, Lanius RA, Nievergelt CM, et al. Post-traumatic stress disorder. Nat Rev Dis Primers (2015) 1:15057. doi: 10.1038/nrdp.2015.57

2. Yaşan A, Güzel A, Tamam Y, Ozkan M. Predictive factors for acute stress disorder and posttraumatic stress disorder after motor are affected by the present-moment cognitive activities $(17,60)$. As the number of resting-state $\mathrm{dFC}$ studies grows, we may gain a better understanding of these properties and their relation to the psychopathology. Secondly, since the dFC based on the sliding-window approach is composed of a few time points, the dynamic analysis is particularly sensitive to the physiological noise (61). Although, we did not denoised the physiological noise individually, we denoised the physiological noise in the preprocessing steps and group-level test and we also chose a relatively large window size in order to diminish the adverse effects of physiological noise. Thirdly, we only examined significant differences in regions exhibiting abnormal dynamic activity to focusing on the dynamic pattern related to PTSD; further exploration of static results is needed in our future works. Fourthly, the correlation results did not survive the FWE correction, so further exploration of the abnormal dynamic patterns and CAPS subscales is needed in our future large sample research to evaluate the relations between the dynamic patterns and specific clinical symptoms severity, such as intrusive memory and flash back.

In conclusion, this resting-state $\mathrm{dFC}$ (combine the dReHo and $\mathrm{dFC}$ ) study provided evidence that the PTSD patients exhibited aberrant dReHo and $\mathrm{dFC}$ in comparison with the HC. Decreased variability within the DMN may suggest dysfunction of self-referential processing in PTSD patients, while increased variability between the insula and $\mathrm{PCu}$ may suggest dysregulation between the DMN and the SN.

\section{ETHICS STATEMENT}

Permission to undertake this study was granted by the ethics committee of Guangdong Second Provincial General Hospital.

\section{AUTHOR CONTRIBUTIONS}

SF designed the experiment. SF and XM carried out the experiment. ZB collected and sorted out the data. YW, MLiu, YY, ZL, SH, MLi, and KH helped on data management and processing. SF and GJ wrote the manuscript.

\section{SUPPLEMENTARY MATERIAL}

The Supplementary Material for this article can be found online at: https://www.frontiersin.org/articles/10.3389/fpsyt.2019.00234/ full\#supplementary-material 
independent component analysis. Cell Physiol Biochem (2018) 48:99-110. doi: $10.1159 / 000491666$

5. Zhang Y, Xie B, Chen H, Li M, Liu F, Chen H. Abnormal functional connectivity density in post-traumatic stress disorder. Brain Topogr (2016) 29:1-7. doi: 10.1007/s10548-016-0472-8

6. Akiki TJ, Averill CL, Wrocklage KM, Scott JC, Averill LA, Schweinsburg B, et al. Default mode network abnormalities in posttraumatic stress disorder: a novel network-restricted topology approach. NeuroImage (2018) 176:1-27. doi: 10.1016/j.neuroimage.2018.05.005

7. Bluhm RL, Williamson PC, Osuch EA, Frewen PA, Stevens TK, Boksman $\mathrm{K}$, et al. Alterations in default network connectivity in posttraumatic stress disorder related to early-life trauma. J Psychiatry Neurosci (2009) 34:187-94. http://jpn.ca/vol34-issue3/34-3-187/

8. Lanius RA, Bluhm RL, Coupland NJ, Hegadoren KM, Rowe B, Théberge J, et al. Default mode network connectivity as a predictor of post-traumatic stress disorder symptom severity in acutely traumatized subjects. Acta Psychiatr Scand (2009) 121:33-40. doi: 10.1111/j.1600-0447.2009.01391.x

9. Koch SBJ, van Zuiden M, Nawijn L, Frijling JL, Veltman DJ, Olff M. Aberrant resting-state brain activity in posttraumatic stress disorder: a meta-analysis and systematic review. Depress Anxiety (2016) 33:592-605. doi: 10.1002/da.22478

10. Biswal BB. Resting state fMRI: a personal history. NeuroImage (2012) 62:938-44. doi: 10.1016/j.neuroimage.2012.01.090

11. Chang C, Glover GH. Time-frequency dynamics of resting-state brain connectivity measured with fMRI. NeuroImage (2010) 50:81-98. doi: 10.1016/j.neuroimage.2009.12.011

12. Hutchison RM, Womelsdorf T, Gati JS, Everling S, Menon RS. Resting-state networks show dynamic functional connectivity in awake humans and anesthetized macaques. Hum Brain Mapp (2012) 34:2154-77. doi: 10.1002/ hbm.22058

13. Keilholz SD, Magnuson ME, Pan W-J, Willis M, Thompson GJ. Dynamic properties of functional connectivity in the rodent. Brain Connect (2013) 3:31-40. doi: 10.1089/brain.2012.0115

14. Deco G, Ponce-Alvarez A, Mantini D, Romani GL, Hagmann P, Corbetta M. Resting-state functional connectivity emerges from structurally and dynamically shaped slow linear fluctuations. J Neurosci (2013) 33:11239-52. doi: 10.1523/JNEUROSCI.1091-13.2013

15. Liu F, Wang Y, Li M, Wang W, Li R, Zhang Z, et al. Dynamic functional network connectivity in idiopathic generalized epilepsy with generalized tonic-clonic seizure. Hum Brain Mapp (2017) 38:957-73. doi: 10.1002/hbm.23430

16. Handwerker DA, Roopchansingh V, Gonzalez-Castillo J, Bandettini PA. Periodic changes in fMRI connectivity. NeuroImage (2012) 63:1712-9. doi: 10.1016/j.neuroimage.2012.06.078

17. Kaiser RH, Whitfield-Gabrieli S, Dillon DG, Goer F, Beltzer M, Minkel J, et al. Dynamic resting-state functional connectivity in major depression. Neuropsychopharmacology (2016) 41:1822-30. doi: 10.1038/npp.2015.352

18. Chang C, Metzger CD, Glover GH, Duyn JH, Heinze H-J, Walter M. Association between heart rate variability and fluctuations in resting-state functional connectivity. NeuroImage (2013) 68:93-104. doi: 10.1016/j. neuroimage.2012.11.038

19. Thompson GJ, Magnuson ME, Merritt MD, Schwarb H, Pan W-J, McKinley A, et al. Short-time windows of correlation between large-scale functional brain networks predict vigilance intraindividually and interindividually. Hum Brain Mapp (2012) 34:3280-98. doi: 10.1002/hbm.22140

20. Gross T, Blasius B. Adaptive coevolutionary networks: a review. J R Soc Interface (2008) 5:259-71. doi: 10.1098/rsif.2007.1229

21. Guo W-B, Liu F, Xue Z-M, Yu Y, Ma C-Q, Tan C-L, et al. Abnormal neural activities in first-episode, treatment-naive, short-illness-duration, and treatment-response patients with major depressive disorder: a resting-state fMRI study. J Affect Disord (2011) 135:326-31. doi: 10.1016/j.jad.2011.06.048

22. Liu F, Hu M, Wang S, Guo W, Zhao J, Li J, et al. Abnormal regional spontaneous neural activity in first-episode, treatment-naive patients with late-life depression: a resting-state fMRI study. Prog Neuropsychopharmacol Biol Psychiatry (2012) 39:326-31. doi: 10.1016/j.pnpbp.2012.07.004

23. Zang Y, Jiang T, Lu Y, He Y, Tian L. Regional homogeneity approach to fMRI data analysis. NeuroImage (2004) 22:394-400. doi: 10.1016/j. neuroimage.2003.12.030

24. Zuo X-N, Xu T, Jiang L, Yang Z, Cao X-Y, He Y, et al. Toward reliable characterization of functional homogeneity in the human brain: preprocessing, scan duration, imaging resolution and computational space. NeuroImage (2013) 65:374-86. doi: 10.1016/j.neuroimage.2012.10.017

25. Hudetz AG, Liu X, Pillay S. Dynamic repertoire of intrinsic brain states is reduced in propofol-induced unconsciousness. Brain Connect (2015) 5:10-22. doi: 10.1089/brain.2014.0230

26. Deng L, Sun J, Cheng L, Tong S. Characterizing dynamic local functional connectivity in the human brain. Sci Rep (2016) 6:26976. doi: 10.1038/ srep26976

27. Kim JI, Yoo JH, Kim D, Jeong B, Kim B-N. The effects of GRIN2B and DRD4 gene variants on local functional connectivity in attention-deficit/ hyperactivity disorder. Brain Imaging Behav (2017) 12:247-57. doi: 10.1007/ s11682-017-9690-2

28. Qiu L, Xia M, Cheng B, Yuan L, Kuang W, Bi F, et al. Abnormal dynamic functional connectivity of amygdalar subregions in untreated patients with first-episode major depressive disorder. JPN (2018) 43:262-72. doi: 10.1503/ jpn. 170112

29. Damaraju E, Allen EA, Belger A, Ford JM, McEwen S, Mathalon DH, et al. Dynamic functional connectivity analysis reveals transient states of dysconnectivity in schizophrenia. Neuroimage Clin (2014) 5:298-308. doi: 10.1016/j.nicl.2014.07.003

30. Fu Z, Tu Y, Di X, Du Y, Pearlson GD, Turner JA, et al. Characterizing dynamic amplitude of low-frequency fluctuation and its relationship with dynamic functional connectivity: an application to schizophrenia. NeuroImage (2017) 180:1-13. doi: 10.1016/j.neuroimage.2017.09.035

31. Calhoun VD. Dynamic connectivity states estimated from resting fMRI identify differences among schizophrenia, bipolar disorder, and healthy control subjects. Front Hum Neurosci (2014) 8:897-13. doi: 10.3389/ fnhum.2014.00897

32. Andrews-Hanna JR, Smallwood J, Spreng RN. The default network and self-generated thought: component processes, dynamic control, and clinical relevance. Ann N Y Acad Sci (2014) 1316:29-52. doi: 10.1111/nyas.12360

33. Kennis M, van Rooij SJH, van den Heuvel MP, Kahn RS, Geuze E. Functional network topology associated with posttraumatic stress disorder in veterans. Neuroimage Clin (2016) 10:302-9. doi: 10.1016/j.nicl.2015.12.008

34. Miller DR, Logue MW, Wolf EJ, Maniates H, Robinson ME, Hayes JP, et al. Posttraumatic stress disorder symptom severity is associated with reduced default mode network connectivity in individuals with elevated genetic risk for psychopathology. Depress Anxiety (2017) 34:632-40. doi: 10.1002/da.22633

35. Patriat R, Birn RM, Keding TJ, Herringa RJ. Default-mode network abnormalities in pediatric posttraumatic stress disorder. J Am Acad Child Adolesc Psychiatry (2016) 55:319-27. doi: 10.1016/j.jaac.2016.01.010

36. Liu F, Xie B, Wang Y, Guo W, Fouche J-P, Long Z, et al. Characterization of post-traumatic stress disorder using resting-state fMRI with a multi-level parametric classification approach. Brain Topogr (2014) 28:221-37. doi: 10.1007/s10548-014-0386-2

37. Jin C, Jia H, Lanka P, Rangaprakash D, Li L, Liu T, et al. Dynamic brain connectivity is a better predictor of PTSD than static connectivity. Hum Brain Mapp (2017) 38:4479-96. doi: 10.1002/hbm.23676

38. Preti MG, Bolton TA, Van De Ville D. The dynamic functional connectomestate-of-the-art and perspectives. NeuroImage (2016) 160:1-33. doi: 10.1016/j.neuroimage.2016.12.061

39. Blake DD, Weathers FW, Nagy LM, Kaloupek DG, Gusman FD, Charney DS, Keane TM. The development of a Clinician-Administered PTSD Scale. J Trauma Stress (1995) 8:75-90. doi: 10.1007/bf02105408

40. Zung WW. A rating instrument for anxiety disorders. Psychosomatics (1971) 12:371-9. doi: 10.1016/S0033-3182(71)71479-0

41. Zung WW. A Self-Rating Depression Scale. Arch Gen Psychiatry (1965) 12:63-70. doi: 10.1001/archpsyc.1965.01720310065008

42. Jenkinson M, Bannister P, Brady M, Smith S. Improved optimization for the robust and accurate linear registration and motion correction of brain images. NeuroImage (2002) 17:825-41. doi: 10.1006/nimg.2002.1132

43. Yan C-G, Craddock RC, Zuo X-N, Zang Y-F, Milham MP. Standardizing the intrinsic brain: towards robust measurement of inter-individual variation in 1000 functional connectomes. NeuroImage (2013) 88:1-17. doi: 10.1016/j. neuroimage.2013.04.081

44. Leonardi N, Van De Ville D. On spurious and real fluctuations of dynamic functional connectivity during rest. NeuroImage (2015) 104:430-6. doi: 10.1016/j.neuroimage.2014.09.007 
45. Ball T, Breckel TPK, Mutschler I, Aertsen A, Schulze-Bonhage A, Hennig J, et al. Variability of fMRI-response patterns at different spatial observation scales. Hum Brain Mapp (2011) 33:1155-71. doi: 10.1002/ hbm. 21274

46. Pajula J, Tohka J. Effects of spatial smoothing on inter-subject correlation based analysis of FMRI. Magn Reson Imaging (2014) 32:1114-24. doi: 10.1016/j.mri.2014.06.001

47. Leech R, Sharp DJ. The role of the posterior cingulate cortex in cognition and disease. Brain (2013) 137:12-32. doi: 10.1093/brain/awt162

48. Guo W, Liu F, Yao D, Jiang J, Su Q, Zhang Z, et al. Decreased default-mode network homogeneity in unaffected siblings of schizophrenia patients at rest. Psychiatry Res Neuroimaging (2014) 224:218-24. doi: 10.1016/j. pscychresns.2014.08.014

49. Liu F, Guo W, Fouche J-P, Wang Y, Wang W, Ding J, et al. Multivariate classification of social anxiety disorder using whole brain functional connectivity. Brain Struct Funct (2013) 220:101-15. doi: 10.1007/ s00429-013-0641-4

50. Buckner RL, Andrews-Hanna JR, Schacter DL. The brain's default network: anatomy, function, and relevance to disease. Ann N Y Acad Sci (2008) 1124:1-38. doi: 10.1196/annals.1440.011

51. Zhang Y, Liu F, Chen H, Li M, Duan X, Xie B, et al. Intranetwork and internetwork functional connectivity alterations in post-traumatic stress disorder. J Affect Disord (2015) 187:114-21. doi: 10.1016/j.jad.2015. 08.043

52. Cole MW, Repovš G, Anticevic A. The frontoparietal control system. Neuroscientist (2014) 20:652-64. doi: 10.1177/1073858414525995

53. Petersen SE, Fox PT, Posner MI, Mintun M, Raichle ME. Positron emission tomographic studies of the cortical anatomy of single-word processing Nature (1988) 331:585-9. doi: 10.1038/331585a0

54. Yin Y, Li L, Jin C, Hu X, Duan L, Eyler LT, et al. Abnormal baseline brain activity in posttraumatic stress disorder: a resting-state functional magnetic resonance imaging study. Neurosci Lett (2011) 498:185-9. doi: 10.1016/j. neulet.2011.02.069
55. Patel NV, Finch CE. The glucocorticoid paradox of caloric restriction in slowing brain aging. Neurobiol Aging (2002) 23:707-17. doi: 10.1016/ S0197-4580(02)00017-9

56. Uddin LQ. Salience processing and insular cortical function and dysfunction. Nat Rev Neurosci (2015) 16:55-61. doi: 10.1038/nrn3857

57. Menon V, Uddin LQ. Saliency, switching, attention and control: a network model of insula function. Brain Struct Funct (2010) 214:655-67. doi: 10.1007/s00429-010-0262-0

58. Lei D, Li K, Li L, Chen F, Huang X, Lui S, et al. Disrupted functional brain connectome in patients with posttraumatic stress disorder. Radiology (2015) 276:818-27. doi: 10.1148/radiol.15141700

59. Sripada RK, King AP, Welsh RC, Garfinkel SN, Wang X, Sripada CS, et al Neural dysregulation in posttraumatic stress disorder. Psychosom Med (2012) 74:904-11. doi: 10.1097/PSY.0b013e318273bf33

60. Buckner RL, Krienen FM, Yeo BTT. Opportunities and limitations of intrinsic functional connectivity MRI. Nat Neurosci (2013) 16:832-7. doi: 10.1038/nn.3423

61. Hutchison RM, Womelsdorf T, Allen EA, Bandettini PA, Calhoun VD, Corbetta M, et al. Dynamic functional connectivity: promise, issues, and interpretations. NeuroImage (2013) 80:360-78. doi: 10.1016/j. neuroimage.2013.05.079

Conflict of Interest Statement: The authors declare that the research was conducted in the absence of any commercial or financial relationships that could be construed as a potential conflict of interest.

Copyright (c) $2019 \mathrm{Fu}, \mathrm{Ma}, \mathrm{Wu}, \mathrm{Bai}, \mathrm{Yi}$, Liu, Lan, Hua, Huang, Li and Jiang. This is an open-access article distributed under the terms of the Creative Commons Attribution License (CC BY). The use, distribution or reproduction in other forums is permitted, provided the original author(s) and the copyright owner(s) are credited and that the original publication in this journal is cited, in accordance with accepted academic practice. No use, distribution or reproduction is permitted which does not comply with these terms. 